\title{
Pharmacological interventions for obsessive-compulsive personality disorder
}

\author{
Rajesh Alex ${ }^{1}$, Michael Ferriter ${ }^{1}$, Hannah Jones ${ }^{1}$, Nick Huband ${ }^{2}$, Conor Duggan ${ }^{2}$, Birgit A \\ Völlm ${ }^{2}$, Jutta Stoffers ${ }^{3}$, and Klaus Lieb ${ }^{4}$ \\ ${ }^{1}$ Literature and Evidence Research Unit (LERU), Institute of Mental Health, Nottinghamshire \\ Healthcare NHS Trust, Woodbeck, UK \\ ${ }^{2}$ Section of Forensic Mental Health, Institute of Mental Health, Nottingham, UK \\ ${ }^{3}$ Department of Psychiatry and Psychotherapy, University Medical Center Freiburg, Freiburg, \\ Germany \\ ${ }^{4}$ Department of Psychiatry and Psychotherapy, University Medical Center Mainz, Mainz, \\ Germany
}

\begin{abstract}
This is the protocol for a review and there is no abstract. The objectives are as follows:

To evaluate the potential beneficial and adverse effects of pharmacological interventions for people with obsessive-compulsive personality disorder and to make recommendations for future areas of research.
\end{abstract}

\section{BACKGROUND}

\section{Description of the condition}

Obsessive compulsive personality disorder is a subcategory of personality disorder. The current edition of the Diagnostic and Statistical Manual of Mental Disorders (DSM-IV-TR; APA 2000) defines personality disorder as: "an enduring pattern of inner experience and behaviour that deviates markedly from the expectations of the person's culture, is pervasive and inflexible, has an onset in adolescence or early adulthood, is stable over time, and leads to distress or impairment".

Copyright $\odot 2010$ The Cochrane Collaboration. Published by John Wiley $\Delta$ Sons, Ltd.

Contact address: Michael Ferriter, Literature and Evidence Research Unit (LERU), Institute of Mental Health, Nottinghamshire Healthcare NHS Trust, The Clair Chilvers Centre, Rampton Hospital, Woodbeck, Nottinghamshire, DN22 OPD, UK.

Michael.Ferriter@nottshc.nhs.uk.

CONTRIBUTIONS OF AUTHORS RA and MF helped prepare the protocol. NH and JS will examine and select the citations and CD will provide adjudication. BV and SG will examine and select the papers and CD will provide adjudication. RA and HJ will extract the data and MF will adjudicate. HJ and MF will complete the risk of bias assessment and RA will adjudicate. HJ and RA will enter the data. RA, with the help and advice of KL, will write the discussion with additional contributions from the other authors (MF, $\mathrm{CD}, \mathrm{NH}, \mathrm{HJ}$ JS, BV, SG).

Editorial group: Cochrane Developmental, Psychosocial and Learning Problems Group.

Publication status and date: New, published in Issue 5, 2010.

DECLARATIONS OF INTEREST None. 
Obsessive-compulsive personality disorder is identified by traits that include perfectionism, rigidity and stubbornness, and miserliness. It is diagnosed, according to DSM-IV-TR (APA 2000), as follows. Individuals demonstrate a pervasive pattern of preoccupation with orderliness, perfectionism, and mental and interpersonal control, at the expense of flexibility, openness and efficiency, beginning by early adulthood and present in a variety of contexts, as indicated by four (or more) of the following:

1. is preoccupied with details, rules, lists, order, organisation or schedules to the extent that the major point of the activity is lost;

2. shows perfectionism that interferes with task completion (e.g. is unable to complete a project because his or her own overly strict standards are not met);

3. is excessively devoted to work and productivity to the exclusion of leisure activities and friendships (not accounted for by obvious economic necessity);

4. is over conscientious, scrupulous and inflexible about matters of morality, ethics or values (not accounted for by cultural or religious identification);

5. is unable to discard worn-out or worthless objects even when they have no sentimental value;

6. is reluctant to delegate tasks or to work with others unless they submit to exactly his or her way of doing things;

7. adopts a miserly spending style toward both self and others; money is viewed as something to be hoarded for future catastrophes;

8. shows rigidity and stubbornness.

The tenth revision of the International Classification of Diseases (ICD-10) refers to this disorder as 'anankastic personality' (WHO 1992).

According to DSM-IV-TR (APA 2000) the prevalence of obsessive-compulsive personality disorder has been estimated from community samples to be around $1 \%$ and from clinical samples around $3 \%$ to $10 \%$. Samuels 2002 calculated the prevalence of obsessivecompulsive personality disorder using DSM-IV criteria in a community sample to be $1.2 \%$ (Samuels 2002). As is the case with many other personality disorders, the prevalence of obsessive-compulsive personality disorder is generally higher in clinical populations (Zimmerman 2005). Those most likely to receive a diagnosis are white, married, employed males (Nestadt 1991).Nigg 1994 noted in their reviews that while evidence concerning the inheritance of obsessive-compulsive personality disorder is mixed, some research suggests that trait obsessiveness in the normal range is moderately heritable. The condition is associated with other Axis II personality disorders such as paranoid, avoidant and borderline personality disorder (Pfohl B 1995). However, Zimmerman 2005 found an elevated odds ratio of comorbidity with obsessive compulsive personality disorder for paranoid, schizoid and narcissistic but not borderline personality disorder. A common question in the literature on obsessive-compulsive personality disorder concerns the nature of its relationship to the similarly named obsessive-compulsive disorder (OCD) (DSM-IV and ICD-10). The classic distinction between these disorders is that obsessions and compulsions in OCD are thought 
to be egodystonic (i.e. perceived as originating from outside the self or unacceptable to the self) whereas obsessive-compulsive personality disorder character traits are thought to be ego-syntonic (i.e. perceived as originating from within the self and consistent with and acceptable to the self) (Pollak 1987; Stein 1993). These boundaries are not always firm, however these two disorders are generally regarded as separate and distinct (Stein 1993).

According to DSM-IV-TR (APA 2000) individuals with anxiety disorders, including social phobia and specific phobia, have an increased likelihood of meeting the criteria for obsessive-compulsive personality disorder. In addition, OCD and eating disorders, anorexia nervosa in particular, have received special attention regarding their relationship to obsessive-compulsive personality disorder. Some research suggests that obsessivecompulsive personality disorder traits may predispose people to develop an eating disorder. For example, studies investigating the co-morbidity between obsessive-compulsive personality disorder and eating disorders have yielded estimates ranging from $3.3 \%$ to $60 \%$ (Serpell 2002).

Finally, personality disorders such as obsessive-compulsive personality disorder are also a significant source of psychiatric morbidity, accounting for more impairment in functioning than major depressive disorder alone (Skodol 2002).

\section{Description of the intervention}

It has been argued that people with personality disorders may respond to pharmacological interventions that target both their state and trait symptoms. This highlights the need to evaluate drug treatments that target the cognitive-perceptual, affective, impulsivebehavioural and anxious-fearful domains of personality disorder (Soloff 1998).

A number of authors have reviewed the evidence relating to treatment of personality disorders with antidepressants, benzodiazepines, anticonvulsants, psychostimulants, antipsychotics and mood stabilisers (Stein 1992; Warren 2001). Although there are no specific medications for the treatment of obsessive-compulsive personality disorder, antidepressants, including tricyclic antidepressants, and anticonvulsant medications, may be helpful. Stein 1993 reported anecdotal evidence of the efficacy of serotonergic agents in patients with obsessive-compulsive personality disorder. Overall, these reviews found the evidence base for pharmacological interventions for obsessive-compulsive personality disorder to be weak, since the bulk of the studies reviewed focused on individuals with borderline personality disorders or on individuals with OCD. It is also important to consider all relevant studies without restriction on pharmaceutical agents, and to consider pharmacological interventions where drugs are given not only as monotherapy but also as an adjunctive intervention.

\section{How the intervention might work}

Several arguments have been put forward justifying drug treatment for personality disorders (Tyrer 2004) and there is a growing body of evidence that personality disorders are associated with neurochemical abnormalities, whether inherited or arising out of physical or psychological trauma (Coccaro 1998; Skodol 2002). One justification for the use of pharmacotherapy is that it has potential to correct deficits or imbalances in certain 
neurotransmitter systems, and so may be able to restore the central nervous systems of people with personality disorder to a more normal neurochemical state (Markovitz 2004).

Several neurochemical abnormalities appear associated with obsessive-compulsive personality disorder, which suggests a role for pharmacological interventions. For example, Greve 2002 reported anecdotal evidence for the anticonvulsant carbamazepine in the treatment of irritability and hostility associated with obsessive-compulsive personality disorder.

An alternative approach (Soloff 1998) is the idea that the likely impact of drugs on the primary symptoms in personality disorder can broadly be predicted from drug effects when used in Axis I disorders. In practice there are reports of behavioural dyscontrol improving in response to lithium (Links 1990) and to anticonvulsants such as carbamazepine (Cowdry 1989), sodium valproate (Stein 1995) and divalproex sodium (Wilcox 1995).

\section{Why it is important to do this review}

Obsessive-compulsive personality disorder is an important condition that has a considerable impact on individuals and families. Personality disorders are a significant source of psychiatric morbidity, accounting for more impairment in functioning than major depressive disorder (Skodol 2002). In addition, Newton-Howes 2006 reported that combined depression and personality disorder is associated with a poorer outcome than depression alone. This is a neglected area of research, as pointed out by Grilo 2004. There has been increased interest in developing and evaluating pharmacological treatments for personality disorder in recent years, suggesting that a systematic review is now timely.

\section{OBJECTIVES}

To evaluate the potential beneficial and adverse effects of pharmacological interventions for people with obsessive-compulsive personality disorder and to make recommendations for future areas of research.

\section{METHODS}

\section{Criteria for considering studies for this review}

Types of studies-Controlled trials in which participants have been randomly allocated to an experimental group and a placebo control group. We will include all relevant randomised controlled trials, with or without blinding.

Types of participants-Men or women, 18 years or over, with a diagnosis of obsessivecompulsive personality disorder defined by any operational criteria, such as DSM-IV-TR (APA 2000). We will include studies of people diagnosed with co-morbid personality disorders or other mental health problems, other than the major functional mental illnesses (i.e. schizophrenia, schizoaffective disorder or bipolar disorder). The decision to exclude persons with co-morbid major functional illness is based on the rationale that the presence of such disorders (and the possible confounding effects of any associated management or 
treatment) might obscure whatever other psychopathology (including personality disorder) might be present.

Types of interventions-People with personality disorders may respond to pharmacological interventions that target both their state and trait symptoms. Although it has been argued that drug treatments that target the cognitive-perceptual, affective, impulsivebehavioural and anxious-fearful domains of personality disorder need to be evaluated (Soloff 1998), we will carry out the review without any a priori assumptions about the effectiveness of certain drugs in a specific domain. We will include in the review studies of any drug(s) with psychotropic properties, including those falling within the following classes of pharmacological interventions (as defined by the BNF 2009):

1. hypnotics, anxiolytics and barbiturates;

2. antipsychotic drugs (including depot injections), antimanic drugs;

3. antidepressant drugs(tricyclic and related, monoamineoxidase inhibitors, SSRIs and related, and other antidepressant drugs);

4. central nervous system stimulants;

5. antiepileptics/mood stabilising agents;

6. drugs used in essential tremor, chorea, tics and related disorders;

7. drugs used in substance dependence;

8. dopaminergic drugs used in parkinsonism;

9. others.

We will include studies evaluating a combination of drug interventions. We will include studies where there is an additional intervention (pharmacological or psychological) if the additional intervention is administered for all arms of the trial, e.g. pharmacological intervention plus cognitive behavioural therapy (CBT) versus placebo plus CBT, or antidepressant plus anticonvulsant versus placebo plus anticonvulsant.

Types of outcome measures-Primary and secondary outcomes are listed below in terms of single constructs. We anticipate that a range of outcome measures will have been used in the studies included in the review (for example, obsession may be measured by a self-report instrument or by an external observer). We will included outcomes that are measured at post-treatment or follow-up periods (or both) of up to six months, six to 12 months, and more than 12 months.

\section{Primary outcomes:}

- Recovery, as measured by obsessive-compulsive symptom levels. We will accept validated clinician-rated scales such as the National Institute of Mental Health Obsessive-Compulsive Scale (NIMH-OCS) (CCSG 1991), or self-rating scales such as the Yale-Brown Obsessive Compulsive Scale (Y-BOCS) (Goodman 1989) and the Maudsley Obsessive Compulsive Inventory (MOCI) (Hodgson 1977). 
- Adverse events, measured as incidence of overall adverse events and of the three most common adverse events(dichotomous outcome, measured as numbers reporting).

In addition we will use the following outcome measures to assess social functioning:

- Global state/functioning (continuous outcome), measured through improvement on the Global Assessment of Functioning numeric scale (GAF; APA 2000) or Clinical Global Impression of Severity scale (CGI-S).

- Social functioning(continuous outcome), measured through improvement in scores on the Social Adjustment Scale (SAS-SR; Weissman 1976), the Social Functioning Questionnaire (SFQ; Tyrer 2005) or a similar validated instrument or scale producing a composite score of severity of overall burden, such as the Symptom Checklist-90 (revised) (SCL-90R).

- Quality of life, using measures such as the SF36 (Ware 1993) or European Quality of Life instrument (EuroQoL Group 1990)).

\section{Secondary outcomes:}

- Participant discontinuation rates, starting from the point of randomised allocation.

- Depressive symptoms (using validated scales such as the Hamilton Depression Rating Scale (HAMD) (Hamilton 1969) and the Beck Depression Inventory (BDI) (Beck 1961).

- Anxiety symptoms (using validated scales such as the Hamilton Anxiety Rating Scale (HAMA) (Hamilton 1959), the State-Trait Anxiety Inventory (STAI) (Spielberg 1983) and the Beck Anxiety Inventory (BAI) (Beck 1988).

- Absence of treatment response (score of 'not improved' or 'little improved') or treatment response (score of 'very much improved' or 'much improved' on all scales).

- Satisfaction with treatment (using the Client Satisfaction Questionnaire (CSQ-8, Attkisson 1982) or similar validated instruments).

\section{Search methods for identification of studies}

Electronic searches-We will search the following electronic databases: the Cochrane Developental, Psychosocial and Learning problems Group Register, the Cochrane Central Register of Controlled Trials (CENTRAL) (The Cochrane Library, current issue); MEDLINE (1950 to current); EMBASE (1980 to current); CINAHL (1982 to current); PsycINFO (1872 to current); the Cochrane Schizophrenia Group register of trials related to Forensic Mental Health; ASSIA; BIOSIS; COPAC; Dissertation Abstracts; IBSSISIProceedings; ISI-SCI (Science Citation Index); ISI-SSCI (Social Sciences Citation Index); OpenSIGLE; Sociological Abstracts; ZETOC; UK Clinical Trials Gateway*; ClinicalTrials.gov*; Action Medical Research*; King's College London (UK)*; ISRCTN Register*; The Wellcome Trust Register*; NHS Trusts Clinical Trials Register*; NHS R $\Delta D$ Health Technology Assessment Programme Register (HTA)*; and NHS R $\Delta$ D Regional 
Programmes Register* (*will be searched using the metaRegister of Controlled Trials (http://www.controlled-trials.com/mrct/)).

The search terms for MEDLINE are shown Appendix 1. We will search MEDLINEin combination with the Cochrane Collaboration's search strategy for identifying reports of controlled trials as detailed in Section 6.4.11 of the Cochrane Handbook for Systematic Reviews of Interventions (Higgins 2008). Similar strategies to identify participants and controlled trials will be developed for the other databases.

\section{Searching other resources}

Handsearching: We will search the reference lists of included and excluded studies for additional relevant trials. We will also examine bibliographies of systematic review articles published in the last five years to identify relevant studies.

Requests for additional data: We will contact authors of relevant studies to enquire about other sources of information and the first author of each included study for information regarding unpublished data. We will contact a representative from all major pharmaceutical companies to request information about any published/unpublished trials.

\section{Data collection and analysis}

Selection of studies-This review is part of a larger series of reviews of personality disorders, therefore we will carry out selection of studies in two stages. In the first stage, two authors (JS and $\mathrm{NH}$ ) will read titles and abstracts independently by and assess these against the inclusion criteria to identify all studies carried out in participants with personality disorder, regardless of any specific personality disorder(s) diagnosed. In the second stage, two independent authors (BV and SG) will assess full copies of studies identified in stage one against the inclusion criteria. This second stage assessment will identify not only trials with participants diagnosed with obsessive-compulsive personality disorder, but also trials with participants having a mix of personality disorders for which data on a subgroup with obsessive-compulsive personality disorder may be available.

We will resolve uncertainties concerning the appropriateness of studies for inclusion in the review through consultation with a third author (CD). Authors will not be blinded to the name(s) of the study author(s), their institution(s) or publication sources at any stage of the review.

Data extraction and management-Two authors (HJ and RA) will extract data independently using a data extraction form. We will enter data into RevMan 5 (RevMan 2008). Where data are not available in the published trial reports, we will contact the authors and ask them to supply the missing information.

Assessment of risk of bias in included studies-For each included study, two authors (HJ and MF) will independently complete the Cochrane Collaboration's tool for assessing risk of bias (Higgins 2008, section 8.5.1). Any disagreement will be resolved through consultation with a third author (RA). We will assess the degree to which: 
- the allocation sequence was adequately generated (sequence generation);

- the allocation was adequately concealed (allocation concealment);

- knowledge of the allocated interventions was adequately prevented during the study (blinding);

- incomplete outcome data were adequately addressed;

- reports of the study were free of suggestion of selective outcome reporting; and

- the study was apparently free of other problems that could put it at high risk of bias.

We will allocate each domain one of three possible categories for each of the included studies: 'Yes' for low risk of bias, 'No' for high risk of bias, and 'Unclear' where the risk of bias is uncertain or unknown.

Measures of treatment effect-For dichotomous (binary) data, we will use the odds ratio (OR) with a 95\% confidence interval (CI) to summarise results within each study. The odds ratio is chosen because it has statistical advantages relating to its sampling distribution and its suitability for modelling, and it is a relative measure so can be used to combine studies. For continuous data, such as the measurement of impulsiveness and aggression on a scale, we will compare the mean score for each outcome as determined by a standardised tool between the two groups to give a mean difference (MD), again with a 95\% CI. Where possible, we will make these comparisons at specific follow-up periods: (1) within the first month, (2) between one and six months, and (3) between six and 12 months. Where possible, we will present endpoint data. Where both endpoint and change data are available for the same outcomes, then we will only report the former.

We will report continuous data that are skewed in a separate table, and will not calculate treatment effect sizes to minimise the risk of applying parametric statistics to data that depart significantly from a normal distribution. We define skewedness as occurring when, for a scale or measure with positive values and a minimum value of zero, the mean is less than twice the standard deviation (Altman 1996).

We will use the weighted mean difference (WMD) where the same outcome measures are reported in more than one study. We will use the standardised mean difference (SMD) where different outcome measures of the same construct are reported.

\section{Unit of analysis issues}

Cluster-randomised trials: Where trials have used cluster-randomisation, we anticipate that study investigators will have presented their results after appropriately controlling for clustering effects (robust standard errors or hierarchical linear models). If it is unclear whether a cluster-randomised trial has used appropriate controls for clustering, we will contact the study investigators for further information. Where appropriate controls were not used, we will request individual participant data and re-analysed data using multilevel models which control for clustering. Following this, we will meta-analyse effect sizes and standard errors in RevMan using the generic inverse method (Higgins 2008). If appropriate controls were not used and individual participant data are not available, we will seek 
statistical guidance from the relevant Cochrane Methods Group and external experts as to which method to apply to the published results in attempt to control for clustering. If there is insufficient information to control for clustering, we will enter outcome data into RevMan using individuals as the units of analysis, and then use sensitivity analysis to assess the potential biasing effects of inadequately controlled clustered trials (Donner 2001).

Cross-over trials: When conducting a meta-analysis combining the results of cross-over trials, we will use the inverse variance methods recommended by Elbourne (Elbourne 2002). Where data presented from a cross-over trial are restricted (and more information is not available from the original investigators) we will use the presented data within the first phase only, up to the point of cross-over.

Multi-arm trials: We will include all eligible outcome measures for all trial arms in this review. Where there are more than two arms of the trial that meet the inclusion criteria, two or more intervention arms and/or two or more control group arms, there are a number approaches that can be used. These include combining arms to create one single pair-wise comparison; selecting one pair for comparison only; splitting the 'shared' group into two or more groups to provide two or more comparisons; including two or more correlated comparisons or undertaking a multiple-treatment meta-analysis. In the event of a multi-arm trial meeting our inclusion criteria we will seek statistical advice on which of these options is the most appropriate for the study.

Dealing with missing data-We will contact the original investigators to request any missing data and information on whether or not data can be assumed to be missing at random.

For dichotomous data we will report missing data and drop-outs for each included study and will report the number of participants who are included in the final analysis as a proportion of all participants in each study. We will provide reasons for missing data in the narrative summary and will assess the extent to which the results of the review could be altered by the missing data by, for example, a sensitivity analysis based on consideration of 'best-case' and 'worst-case' scenarios (Gamble 2005). Here, the 'best-case' scenario is that where all participants with missing outcomes in the experimental condition had good outcomes, and all those with missing outcomes in the control condition had poor outcomes, and the 'worstcase' scenario is the converse (Higgins 2008, section 16.2.2).

For missing continuous data, we will provide a qualitative summary. The standard deviations of the outcome measures should be reported for each group in each trial. If these are not given, we will impute standard deviations using relevant data (for example, standard deviations or correlation coefficients) from other, similar studies (Follmann 1992) but only if, after seeking statistical advice, to do so is deemed practical and appropriate.

Where missing data might be assumed to be missing at random then it may be appropriate to analyse only the available data. Where it may not be possible to assume this, i.e. not missing at random, it might be possible to impute missing data with replacement values, for example last observation carried forward. Other methods of imputing missing values with uncertainty 
or using statistical models will only be used on the advice and with the assistance of a statistician.

Assessment of heterogeneity-We will assess the extent of between-trial differences and the consistency of results of any meta-analysis in three ways: by visual inspection of the forest plots, by performing the $\mathrm{Chi}^{2}$ test of heterogeneity (where a significance level less than 0.10 will be interpreted as evidence of heterogeneity), and by examining the $\mathrm{I}^{2}$ statistic (Higgins 2008; section 9.5.2). The $\mathrm{I}^{2}$ statistic describes approximately the proportion of variation in point estimates due to heterogeneity rather than sampling error. We will consider $\mathrm{I}^{2}$ values of less than $40 \%$ as indicating that heterogeneity may not be important, that values in the range $30 \%$ to $60 \%$ may represent moderate heterogeneity, values in the range $50 \%$ to $90 \%$ may represent substantial heterogeneity and that $75 \%$ to $100 \%$ may represent considerable heterogeneity. We will attempt to identify any significant determinants of heterogeneity categorised as moderate or high.

We cannot state in advance what our preferred method of dealing with heterogeneity, if present, will be as this will be contingent on the data. However, we will first check that the data have been correctly entered. There are a number of methods that can be used for handling heterogeneity and these include the following. The studies can be examined to explore the reason for heterogeneity but this is not recommended where there are few studies. A random-effects model could be used. Where a study paper is an outlier, sensitivity analysis can be carried out with and without the study. Other strategies include ignoring the heterogeneity, not performing a meta-analysis and changing the effect measure. We will seek statistical advice before using any of these methods.

Assessment of reporting biases-We will draw funnel plots (effect size versus standard error) to assess publication bias if sufficient studies are found. Asymmetry of the plots may indicate publication bias, although it may also represent a true relationship between trial size and effect size. If such a relationship is identified, we will examine the clinical diversity of the studies further as a possible explanation (Egger 1997).

Data synthesis-We will undertake meta-analyses of the data using both fixed and random-effects models. Meta-analyses may be conducted to combine comparable outcome measures across studies. In carrying out meta-analysis, the weight given to each study will be the inverse of the variance so that the more precise estimates (from larger studies with more events) are given more weight. We will group outcome measures by length of follow up.

Subgroup analysis and investigation of heterogeneity-If sufficient studies are found, we will undertake subgroup analysis to examine the effect on primary outcomes of:

1. participants' principal diagnosis, e.g. personality disorder, eating disorder, OCD;

2. setting (inpatient, custodial, outpatient/community); and

3. class of drug. 
Where we find a number of studies with participants aged less than 18 years, we will perform sensitivity analysis to explore the effect of including/excluding this younger sample.

Sensitivity analysis - If there are sufficient data, we will undertake sensitivity analyses to investigate the robustness of the overall findings in relation to certain study characteristics. We plan a priori sensitivity analyses for:

1. concealment of allocation;

2. blinding of outcome assessors; and

3. extent of drop-outs.

\section{Acknowledgments}

None.

\section{SOURCES OF SUPPORT}

Internal sources

- $\quad$ Nottinghamshire Healthcare NHS Trust, UK.

External sources

- German Federal Ministry of Education and Research Grant no 01KG0812., Germany.

- $\quad$ NHS Cochrane Collaboration Programme Grant Scheme, UK.

\section{Appendix 1. MEDLINE search strategy}

The search terms for MEDLINE will be as follows:

1. exp personality disorders/

2. exp anankastic personality disorder

3. exp obsessive compulsive personality disorder/

4. exp compulsive personality disorder/

5. exp anal character/

6. exp passive-aggressive personality disorder/

7. obsessive

8. compulsive

9. anal retentive or anal character

10. anankastic

11. (DSM and (Axis II)) or/1-11

We will search MEDLINE in combination with the Cochrane Collaboration's search strategy for identifying reports of controlled trials as detailed in Section 6.4.11 of the Cochrane Handbook for Systematic Reviews of Interventions (Higgins 2008). 
We will develop similar strategies to identify participants and controlled trials for the other databases.

\section{HISTORY}

Protocol first published: Issue 5, 2010

\section{Additional references}

Altman 1996 . Altman DG, Bland JM. Detecting skewedness from summary information. BMJ. 1996; 313:1200. [PubMed: 8916759]

APA 2000 . American Psychiatric Association. Diagnostic and Statistical Manual of Mental Disorders - Text Revision (DSM-IV-TR). American Psychiatric Association; Washington DC: 2000.

Attkisson 1982 . Attkisson CC, Zwick R. The client satisfaction questionnaire. Psychometric properties and correlations with service utilization and psychotherapy outcome. Evaluation and Program Planning. 1982; 5:233-7.

Beck 1961 . Beck AT, Ward CH, Mendelson M, Mock J, Erbaugh J. An inventory for measuring depression. Archives of General Psychiatry. 1961; 4:561-71. [PubMed: 13688369]

Beck 1988 . Beck AT, Epstein N, Brown G, Steer RA. An inventory for measuring clinical anxiety: psychometric properties. Journal of Consulting and Clinical Psychology. 1988; 56:893-7. [PubMed: 3204199]

BNF 2009 . Joint Formulary Committee. British National Formulary. BMJ Publishing Group Ltd; London: Mar. 2009

CCSG 1991 . Clomipramine Collaborative Study Group. Clomipramine in the treatment of patients with obsessive-compulsive disorder. Archives of General Psychiatry. 1991; 48:730-8. [PubMed: 1883256]

Coccaro 1998 . Coccaro EF, Kavoussi RJ, Hauger RL, Cooper TB, Ferris CF. Cerebrospinal fluid vasopressin levels: correlates with aggression and serotonin function in personality-disordered subjects. Archives of General Psychiatry. 1998; 55:708-14. [PubMed: 9707381]

Cowdry 1989 . Cowdry RW, Gardner DL. Pharmacotherapy of borderline personality disorder: alprazolam, carbamazepine, trifluoperazine and tranylcypromin. Archives of General Psychiatry. 1989; 45:111-9. [PubMed: 3276280]

Donner 2001 . Donner A, Piaggio G, Villar J. Statistical methods for the meta-analysis of cluster randomization trials. Statistical Methods in Medical Research. 2001; 10(5):325-38. [PubMed: 11697225]

Egger 1997 . Egger M, Davey-Smith G, Schneider M, Minder C. Bias in meta-analysis detected by a simple, graphical test. BMJ. 1997; 315:629-34. [PubMed: 9310563]

Elbourne 2002 . Elbourne DR, Altman DG, Higgins JP, Curtin F, Worthington HV, Vail A. Metaanalyses involving cross-over trials: methodological issues. International Journal of Epidemiology. 2002; 31(1):140-9. [PubMed: 11914310]

EuroQoL Group 1990 . EuroQoL Group. EuroQoL: a new facility for measurement of health-related quality of life. Health Policy. 1990; 16:199-208. [PubMed: 10109801]

Follmann 1992 . Follmann D, Elliot P, Suh I, Cutler J. Variance imputation for overviews of clinical trials with continuous response. Journal of Clinical Epidemiology. 1992; 45(7):769-73. [PubMed: 1619456]

Gamble 2005 . Gamble C, Hollis S. Uncertainty method on best-worst case analysis in a binary meta-analysis. Journal of Clinical Epidemiology. 2005; 58(6):579-88. [PubMed: 15878471]

Goodman 1989 . Goodman WK, Price LH, Rasmussen SA, Mazure C, Fleischmann RL, Hill CL, et al. The Yale-Brown obsessive compulsive scale 1: development, use and reliability. Archives of General Psychiatry. 1989; 46:1006-11. [PubMed: 2684084] 
Greve 2002 . Greve KW, Adams D. Treatment of features of obsessive-compulsive personality disorder using carbamazepine. Psychiatry and Clinical Neurosciences. 2002; 56:207-8. [PubMed: 11952927]

Grilo 2004 . Grilo CM. Factor structure of DSM-IV criteria for obsessive compulsive personality disorder in patients with binge eating disorder. Acta Psychiatrica Scandinavica. 2004; 109:64-8. [PubMed: 14674960]

Hamilton 1959 . Hamilton M. The assessment of anxiety states by rating. British Journal of Medical Psychology. 1959; 32:50-5. [PubMed: 13638508]

Hamilton 1969 . Hamilton M. Standardised assessment and recording od depressive symptoms. Psychiatria, Neuralgia, Neurochirurgia. 1969; 72(2):201-5.

Higgins 2008 . Higgins, JPT.; Green, S., editors. Cochrane Handbook for Systematic Reviews of Interventions Version 5.0.1 [updated September 2008]. The Cochrane Collaboration; 2008. Available from www.cochrane-handbook.org

Hodgson 1977 . Hodgson RJ, Rachman S. Obsessive-compulsive complaints. Behaviour Research and Therapy. 1977; 15:389-95. [PubMed: 612339]

Links 1990 . Links P. Lithium therapy for borderline patients. Journal of Personality Disorders. 1990; 4:173-81.

Markovitz 2004 . Markovitz PJ. Recent trends in the pharmacotherapy of personality disorders. Journal of Personality Disorders. 2004; 18(1):90-101. [PubMed: 15061346]

Nestadt 1991 . Nestadt G, Romanoski AJ, Brown CH, Chahal R, Merchant A, Folstein MF, et al. DSM-III compulsive personality disorder: an epidemiological survey. Psychological Medicine. 1991; 21:461-71. [PubMed: 1876651]

Newton-Howes 2006 . Newton-Howes G, Tyrer P, Johnson T. Personality disorder and the outcome of depression: meta-analysis of published studies. British Journal of Psychiatry. 2006; 188:1320. [PubMed: 16388064]

Nigg 1994 . Nigg JT, Goldsmith HH. Genetics of personality disorders: perspectives from personality and psychopathology research. Psychological Bulletin. 1994; 115:346-80. [PubMed: 8016285]

Pfohl B 1995 . Pfohl, B.; Blum, N. Obsessive compulsive personality disorder, the DSM IV personality disorder. Guilford Press; New York: 1995.

Pollak 1987 . Pollak JM. Relationship of obsessive-compulsive personality to obsessive-compulsive disorder: a review of the literature. Journal of Psychology. 1987; 121:137-48. [PubMed: 3585808]

RevMan 2008 . The Nordic Cochrane Centre, The Cochrane Collaboration. Review Manager (RevMan). 5.0. The Nordic Cochrane Centre, The Cochrane Collaboration; Copenhagen: 2008.

Samuels 2002 . Samuels J, Eaton WW, Bienvenu OJ, Brown CH, Costa PT, Nestadat G. Prevalence and correlates of personality disorders in a community sample. British Journal of Psychiatry. 2002; 180:536-42. [PubMed: 12042233]

Serpell 2002 . Serpell L, Livingstone A, Neiderman M, Lask B. Anorexia nervosa: obsessivecompulsive disorder, obsessive-compulsive personality disorder, or neither? Clinical Psychology Review. 2002; 22:647-69. [PubMed: 12113200]

Skodol 2002 . Skodol AE, Siever LJ, Livesley WJ, Gunderson JG, Pfohl B, Widiger TA. The borderline diagnosis II: biology, genetics and clinical course. Biological Psychiatry. 2002; 51:951-63. [PubMed: 12062878]

Soloff 1998 . Soloff PH. Algorithms for pharmacological treatment of personality dimensions. Symptom-specific treatments for cognitive-perceptual, affective, and impulsive-behavioral dysregulation. Bulletin of the Menninger Clinic. 1998; 62:195-214. [PubMed: 9604516]

Spielberg 1983 . Spielberg, CD.; Gorsuch, RL.; Luchene, R.; Vagg, PR.; Jacobs, GA. Manual for the State-Trait Anxiety Inventory. Consulting Psychologists Press; Palo Alto, CA: 1983.

Stein 1992 . Stein GS. Drug treatment of the personality disorders. British Journal of Psychiatry. 1992; 161:167-84. [PubMed: 1355689]

Stein 1993 . Stein, DJ.; Hollander, E. Obsessive-compulsive related disorders. Vol. Vol. 1. American Psychiatric Press; Washington DC: 1993. The spectrum of obsessive compulsive related disorders; p. 241-62. 
Stein 1995 . Stein DJ, Simeon D, Frenkel M, Islan MN, Hollander E. An open trial of valproate in borderline personality disorder. Journal of Clinical Psychiatry. 1995; 56:506-10. [PubMed: 7592502]

Tyrer 2004 . Tyrer P, Bateman AW. Drug treatment for personality disorders. Advances in Psychiatric Treatment. 2004; 10:389-98.

Tyrer 2005 . Tyrer P, Nur U, Crawford M. The Social Functioning Questionnaire: a rapid and robust measure of perceived functioning. International Journal of Social Psychiatry. 2005; 51:265-75. [PubMed: 16252794]

Ware 1993 . Ware, JE.; Snow, KK.; Kosinski, M.; Gandek, B. SF-36 Health Survey Manual and Interpretation Guide. New England Medical Centre; Boston: 1993. Health Survey Manual and Interpretation Guide.

Warren 2001 . Warren, F.; Preedy-Fayers, K.; McGauley, G.; Pickering, A.; Norton, K.; Geddes, JR., et al. Review of treatments for severe personality disorder. Home Office; London: 2001.

Weissman 1976 . Weissman MM, Bothwell S. Assessment of social adjustment by patient selfreport. Archives of General Psychiatry. 1976; 33:1111-15. [PubMed: 962494]

WHO 1992 . World Health Organization. The ICD-10 Classification of Mental and Behavioural Disorders. 10th Edition. WHO; Geneva: 1992.

Wilcox 1995 . Wilcox JA. Divalproex sodium as a treatment for borderline personality disorder. Annals of Clinical Psychiatry. 1995; 7(1):33-7. [PubMed: 8541935]

Zimmerman 2005 . Zimmerman M, Rothschild L, Chelminski I. The prevalence of DSM-IV personality disorders in psychiatric outpatients. American Journal of Psychiatry. 2005; 162:1911-18. [PubMed: 16199838] * Indicates the major publication for the study 